\title{
Optimal Reliability of a Smart Grid
}

\author{
Vinny Junior FOBA KAKEU*:(D), Alexandre Teplaira BOUM**(D), Camille Franklin MBEY***(D)
}

*Department of Electrical Engineering, ENSET, University of Douala, 1872 Douala-Cameroon

**Department of Electrical Engineering, ENSET, University of Douala, 1872 Douala-Cameroon

***Department of Electrical Engineering, ENSET, University of Douala, 1872 Douala-Cameroon

(kakeuvinny@gmail.com, boumat2002@yahoo.fr, camillembey@yahoo.fr)

¥Vinny Junior FOBA KAKEU; 1872 Douala-Cameroun, Tel: +237 691836176,

Fax:+237691836176, kakeuvinny@gmail.com

Received: 01.04.2021 Accepted:06.06.2021

\begin{abstract}
This work presents the optimal reliability of a smart grid using Particle Swarm Optimization (PSO) and the Monte Carlo analysis. In the electrical grid, reliability defines the permanent supply of energy and the satisfaction of customers. In this paper, the reliability of a smart grid is examined in the baseline scenario consisted of no faults and the scenarios consisted of faults both in the presence or absence of Distributed Generation units (DGs) using co-simulations. The study determines the optimal allocation and size of the DGs during faulty scenarios which was carried out using PSO. This work proposes an OpenDSS-OMNet++ platform applied in the electrical grid and communication network to achieve the smart distribution grid. The aim consists to assess the capacity of the system according to various possibilities and the automation level of the network. The 13-node IEEE system is implemented using the method and the proposed framework. In case of fault, the voltage drop is $45 \%$, the SAIFI is 40 and with optimal allocation of distributed generation, the voltage drop moves to $2.3 \%$, the SAIFI is 0.55 . The energy losses go from $5232686.67 \mathrm{kWh} /$ year to $211076.68 \mathrm{kWh} /$ year, i.e. a difference of nearly $5021609.99 \mathrm{kWh} /$ year. The final results indicate that our algorithm is efficient and reliable and can help companies of energy for future reliability trials and the progression of distributed generation in smart distribution grid.
\end{abstract}

Keywords co-simulation, reliability, smart grid, PSO, distributed generation.

\section{Introduction}

Nowadays, power grids are facing major failures and outages. It is the reason why so many reliability trials are operated by power distributor to verify the availability of electrical energy to the customers. The electrical grid can generate many reliability issues due to diverse devices and materials. It is an opportunity for energy companies to modernize their facilities in order to minimize the operating costs of their network, while increasing the reliability and safety of the network. The transition to the smart grid is necessary with the advancement of new production, automation and communication technologies [1].

The evolution of power grids associated with communication devices has given rise to a new generation of grids called smart grids. Smart grid is the future generation of electrical network which intelligently integrates the actions of producers and consumers connected and communicating together, in order to provide an efficient, sustainable, reliable, safety and economical supply of electricity [2]. According to author [3], the smart grid is considered as self-sufficient system allowing integration of any kind of generation sources in the electrical grid with the target of reliable, safe and sustainable electrical energy to consumers. In a smart grid, there is a bidirectional transmission of electrical energy and information flow to build a dynamic network [4]. Compared to the conventional grid, the smart grid is the next generation of energy distribution networks including thousands of devices of new information and communication technologies [5]. The smart grid brings several benefits for the power network in terms of: reliability by reducing interruptions and disturbances of energy; economy by minimizing electricity consumption; efficiency by reducing the cost of production, distribution and consumption of energy; environment by reducing polluting emissions through the integration of renewable energies; safety by reducing the risk. It provides all necessary devices to control and automate the electrical grid and to ensure an available and reliable energy to the costumers 
connected to the network [6]. The transition to the smart grid can give new opportunities to manage the distribution and the consumption. To implement a smart grid, many elements should be implemented as renewable resources, electrical and hybrid vehicles, dynamics system, communication utilities and internet connection. grid.

Figure 1 shows a representation of a smart distribution

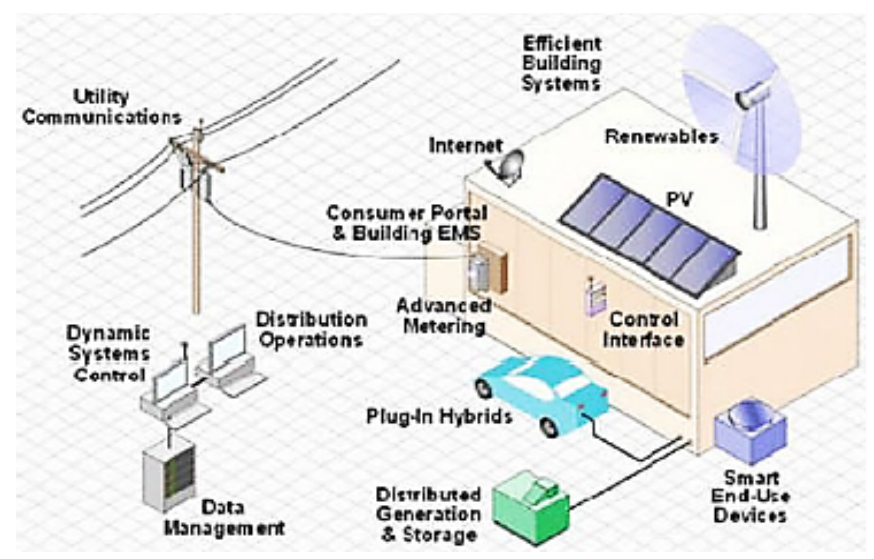

Fig. 1. Representation of a smart grid [7]

The smart grid can integrate Distributed Generation (DG) in order to reduce dependence from fossil resources. Distributed Generation is a new method consisting on renewable power resources which can have a positive impact to the distribution network [8]. Renewable power resources are considered as the main resources for future energy generations due to energy crisis [9]. Distribution Generator can be connected to power generators or directly to the consumption points to provide energy at the loads. In the current electrical network, the electrical power is distributed to loads through lines and electrical elements which can reduce voltage and increase the power losses in the networks. So, the transmission lines are not important when the distributed generators are located near the customers. A good connection of DG can consequently reduce the power losses in the network. Eventually, the integration of distributed generators can be the solution for energy distributor to migrate for a smart, reliable and safe grid [10].

The allocation of DG in distribution system can improve the reliability of the network, reduce the energy losses and minimize the consumption cost for clients. The reliability index can be mathematically estimated as follow:

- SAIDI : is defined as the System Average Interruption Duration Index

$$
\text { SAIDI }=\frac{\sum_{i=0}^{y_{i}}{ }_{i} N_{i}}{N_{s}}
$$

- SAIFI : refers to the System Average Interrupt Frequency Index

$$
S A I F I=\frac{\sum_{i=0}^{n} N_{i}}{N_{s}}
$$

- CAIDI : refers to the Customer Average Interruption Duration Index

$$
\text { CAIDI }=\frac{\text { SAIDI }}{\text { SAIFI }}=\frac{\sum_{i=0}^{n} r_{i} N_{i}}{\sum_{i=0}^{n} N_{i}}
$$

In [11], the author proposed a strategy to select the best type of distributed generation, the optimal location and the size. In this paper, they solved the problem using the valuebased planning method, balancing the cost of installation with the benefits of distributed generation, which include loss of power, reduction of costs, and improvement of reliability. In [12], the author proposed a technique to implement the hybrid optimization algorithm for the location of several DGs with a suitable size. This hybrid optimization method combines the behavior of ant colonies and the cuckoo search algorithm. Distributed generations are ideally installed to minimize energy losses and enhance the voltage profile. The work in [13], presented an optimization algorithm consisting to identify the location and the size of DG in power grid. In this paper, the author simulated the power distribution in OpenDSS driven from Matlab programming. The analysis shows that the appropriate use of DG would significantly reduce energy losses in a network. In [14], the author proposed the multi-leader particle swarm optimization for the optimal allocation and size of distributed generation with the goal to minimize the active losses. The author implemented the method in IEEE 33node test feeder and Malaysian network. In [15], the author presented the analysis of the influence of photovoltaic system in the electrical network. To validate this method, the author used OpenDSS tool implementing five cases in IEEE 30 node system. However, they don't consider the location of PV (Photovoltaic) where voltage drop is bad. In [16], the author studied the intelligent voltage control of distributed generation in distribution system. He tested three methods for optimal penetration of reactive power of Distributed Generation in unbalanced distribution network. To validate these methods, he used 13-node and 123-node system.

To simulate the impact of distributed generation in the grid, some time series tools can offer the best solution for the analysis of the network [17]. The works in the literature applied in conventional networks and they don't really verify the reliability of the electrical grid considering two-way communication.

The contribution of this paper is focusing on a novel smart grid co-simulation through OpenDSS and OMNet++, and also an optimization algorithm using OpenDSS-Matlab platform to address the problem of optimal DGs allocation and sizing. The advantage to first implement the smart grid co-simulation is to provide two-way communication between all components of the electrical grid. The novelty of this paper concern the co-simulation of the smart grid which doesn't exist in the literature. Once the smart grid is built, the integration of distributed generation can be done easily.

This paper is organized as follows. Section 2 presents materials and methods, we will present the software tools used for smartgrid co-simulation and the allocation of DGs. Section 3 shows the results and comparisons for different case study applied on 13-node IEEE system. In section 4, we conclude our paper. 


\section{Materials and methods}

\subsection{Materials}

\subsubsection{The test network}

To perform the network analysis, we will use the 13node IEEE network. The simulation of power flow is implemented using OpenDSS which is detailed in this work. Figure 2 presents the basic structure 13-node IEEE network used for co-simulation. IEEE 13-node network is made of 13 nodes, 12 lines, 1 source, 2 transformers and 15 loads. The source is connected at node 650 . At source substation, the voltage is $115 \mathrm{kV}$ with 3 phases, the frequency is $50 \mathrm{~Hz}$ and the power is $20000 \mathrm{MVA}$. The transformer at the source reduces the voltage to $4.16 \mathrm{kV}$. So, the others nodes are supplied on $4.16 \mathrm{kV}$, excepting the node 634 which is on $0.277 \mathrm{kV}$. A switch is connected between the nodes 671 and 692.

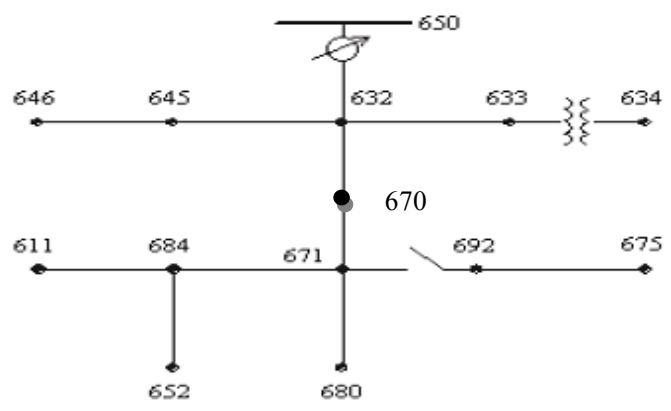

Fig. 2. Basic structure of 13-node IEEE network

The loads connected at the nodes can consume a lot of energy from the source substation and that can conduct to a fault which can happen at node 670. This fault is an overload fault due to excessive consumption of the energy during the year. The effect of this fault is the collapse of the network and interruption of electricity. To avoid the collapse of the network and to reduce the impact of this overload fault, the loads should use the energy from distributed generation units which can significantly reduce the system energy consumed and energy losses.

The co-simulation framework which is proposed, uses OpenDSS version 9.0.0.3 (64-bit), OMNet++ version 4.4 and Matlab R2020a (64-bit). All simulations were carried out on computer icore5, 64 Bit Window 7 system, processor 3.5 GHz, 8 GB RAM.

\subsubsection{OpenDSS}

Open Distribution System Simulator (OpenDSS) is an open source software originally developed (in 1997) by Electrotek Concepts. One of the motivations for the development of this tool was the calculation of harmonics and inter-harmonics on the distribution network. Subsequently, various modules were added, such as Monte Carlo analysis and annual simulations. In 2004, the software was acquired by EPRI, with the aim of providing a tool to simulate advanced automation of power grids. EPRI decided to make the source code freely available on the web site [18]. The initialization of lines, sources and transformers is necessary for modelling the network. OpenDSS works from the command line with its own console. Using the COM server, users can drive OpenDSS from other platforms as Matlab, Excel, Python, VBA and OMNet++. Many network elements as cables, transformers and regulators are contained in OpenDSS. So, the development of large network must be done with attention to avoid any mistake. OpenDSS can be used for network analysis and planning, solar and wind farm simulation and storage, and smart grid simulation. The OpenDSS files are identified with the extension .dss. The results can be .csv or .txt files. OpenDSS can be a solution for energy distributors to prevent any change in their networks [19].

In this paper, OpenDSS is used to solve the network and gives results as power losses, currents, active and reactive powers. Figure 3 shows the structure of OpenDSS.

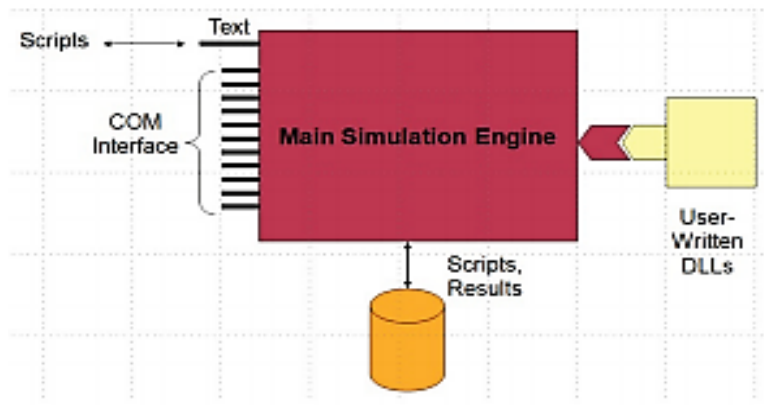

Fig. 3. Structure of OpenDSS

\subsubsection{OMNet++}

Objective Modular Network in $\mathrm{C}++(\mathrm{OMNet}++)$ is a simulation tool written in $\mathrm{C}++$ for communication networks. OMNet ++ has properties for the creation and configuration of models (NED and ini files) ensuring the batch execution and analysis of simulation results while Eclipse provides the $\mathrm{C}++$ editor and other optional elements (UML modelling, access to the database). It allows simulations of an existing real communication network without any risk. The simulation results can be analysed to determine how the actual network may be affected [20].

The communication between OpenDSS and OMNet++ occurs as follow: when a simulation begins for first time, OMNet++ is waiting the information data from OpenDSS. Then, this data is contained in the cache memory. OMNet++ can verify information from the last simulation of OpenDSS contained in the cache memory. A specific simulation time is sent from the simulation machine. The request from OMNet++ reaches the CVR-controller which can run OpenDSS and update the data in the cache memory.

\subsubsection{Matlab}

Matlab (Matrix Laboratory) is a tool based on matrix implementation. Matlab has many toolboxes for a specific work. In this paper, Matlab is used to perform the analysis of the electrical grid after the simulation with OpenDSS.

\subsection{Methods}

\subsubsection{Particle swarm optimization}


Kennedy and Eberhart developed the PSO in 1995. The PSO is meta-heuristic method allowing to optimize an event and a function. The populations are considered as swarm randomly generated. The individuals called as particles are contained in this swarm. The particles have coordinates in the space and can keep the same way based on the best solution. The best solution is achieved amongst all solutions. Figure 4 shows the general structure of PSO particle movement.

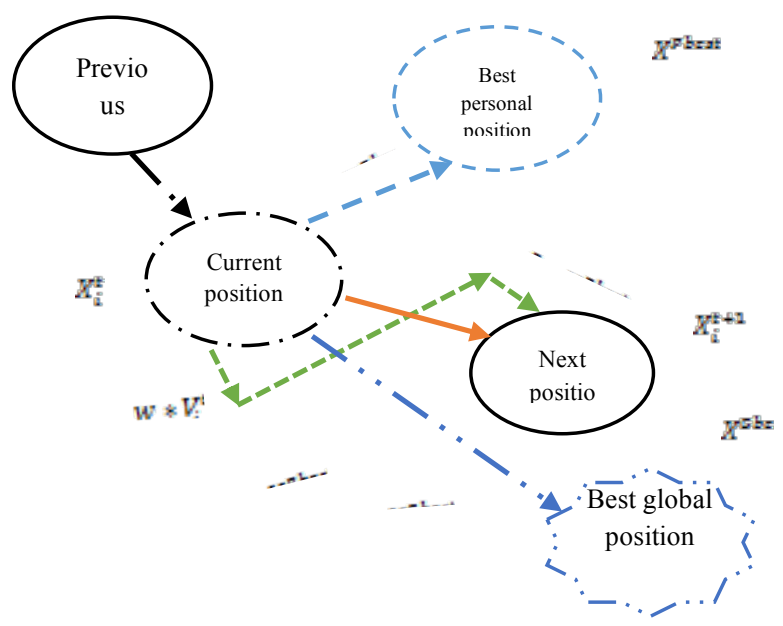

Fig. 4. PSO particle movement

The equation of PSO from figure 4 is shown below:

$V_{i}^{t+1}=\left(w * V_{i}^{t}\right)+\alpha\left(X^{P b e s t}-X_{i}^{t}\right)+\beta\left(X^{G b e s t}-X_{i}^{t}\right)$

$X_{i}^{t+1}=X_{i}^{t}+V_{i}^{t+1}$

With:

$X_{\mathrm{i}}^{\mathrm{t}}$ : Current position of the particle $\mathrm{i}$ at iteration $\mathrm{t}$

$V_{i}^{t}$ : Speed of particle $\mathrm{i}$ on the previous vector

$V_{i}^{t+1}$ speed of particle $i$ at the next position

ws Refers to inertia weight factor

$X^{P b a s t}=\left\{\begin{array}{c}X^{P b a s t(j)} \text { if } O F^{j+1} \geq O F^{j} \\ X_{i}^{t} \text { if } O F^{j+1} \leq O F^{j}\end{array}\right.$

$X^{\text {pbest }}$ : Refers to best personal position of the particle

$X^{G b a s t}=\left\{\begin{array}{l}X^{G b e s t[j]} \text { if } O F^{j+1} \geq O F^{j} \\ X^{p b e s t(j+1)} \text { if } O F^{j+1} \leq O F^{j}\end{array}\right.$

$X^{\text {Gbest: }}$ Best global position of the particle

$\alpha=C_{1} r_{1}$

$\beta=C_{2} r_{2}$

In this paper, we use a novel method consisting to optimize the reliability of the smart grid by a co-simulation and the integration of distributed generation using the PSO algorithm. To optimize the reliability in term of energy losses, voltage drop and SAIFI (System Average Interrupt Frequency Index), the PSO explores the system and can find the best candidate nodes where we can locate DGs. We optimize these values because they reflecting the behavior of the power grid considering various scenarios. This smart algorithm is better comparing with others in the literature.
Figure 5 presents the structure of OpenDSS-Matlab analysis for optimum allocation and size of DGs.

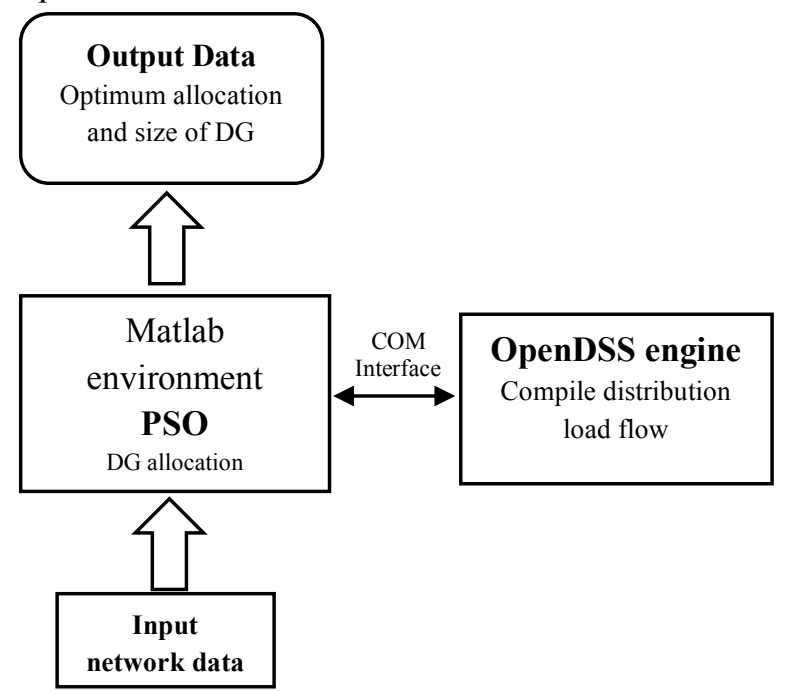

Fig. 5. Structure of OpenDSS-Matlab analysis

Matlab environment is used to drive OpenDSS through the COM interface. Matlab uses the data from OpenDSS to bring an appropriate command. The input data's are the network parameters. The output data of the analysis are the optimum allocation and size of DG in the power grid.

\subsubsection{Annual Monte Carlo simulation}

The process is a Monte Carlo method aimed to assess reliability indices of distribution networks. The simulation can be carried out with or without DG depending the system under study. Input data system parameters, load and generators values and simulation time. In this paper, the simulation is done for one year. The time step is 1 hour with 8760 iterations. Some random variables as current, voltages, failure rates can be generated during the yearly simulation. Then, you can simulate for the first time without failure. The second time you introduce a possibility of failure. The reliability index is generated during this simulation.

\section{Results and Discussion}

\subsection{Smart grid simulation}

Figure 6 shows the data exchange in smart grid using OpenDSS-OMNet++ co-simulation.

It should be noted with this result that, the voltage (V) and the energy consumed (E) are displayed on each bus. The network is controlled from a CVR-Controller. Thus, integrating communication technologies, we obtain a smart grid of the IEEE 13-node system with several smart and communication devices. 


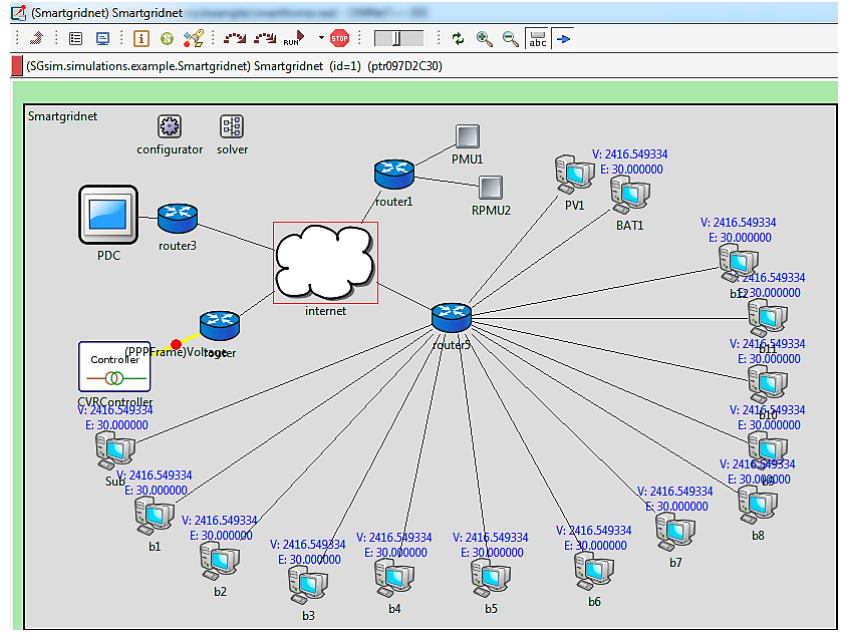

Fig. 6. Smart grid simulation

\subsection{Case without fault}

Figure 7 presents the power flow of test case IEEE 13node system without fault.

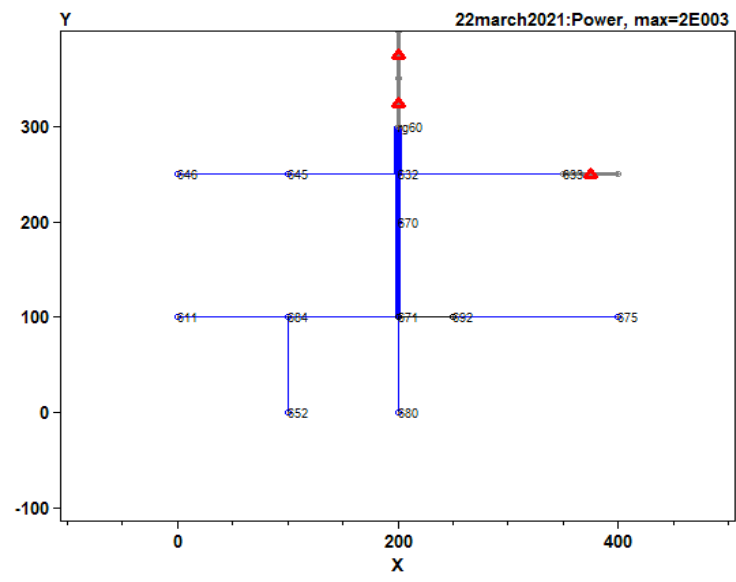

Fig. 7. Power Flow without fault

Figure 8 presents the voltage profile in the system without fault.

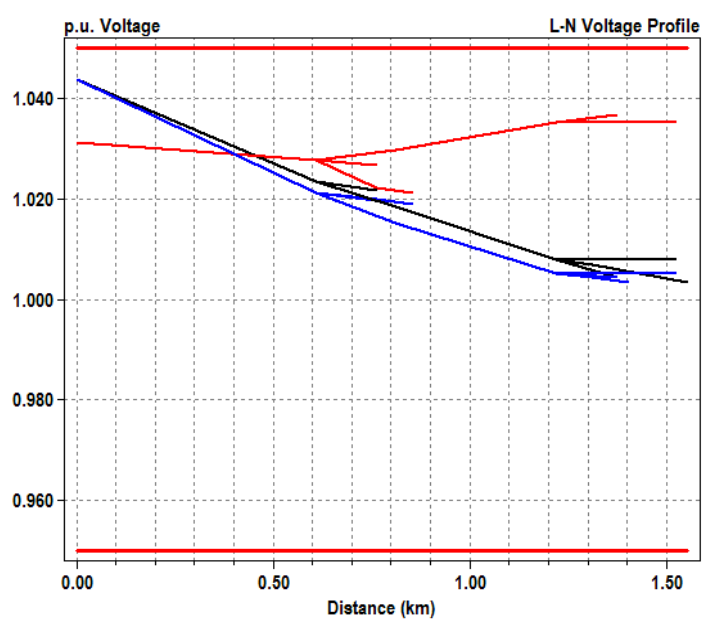

Fig. 8. Voltage profile without fault
Figure 9 presents the voltage profile at node 611 without fault for one year.

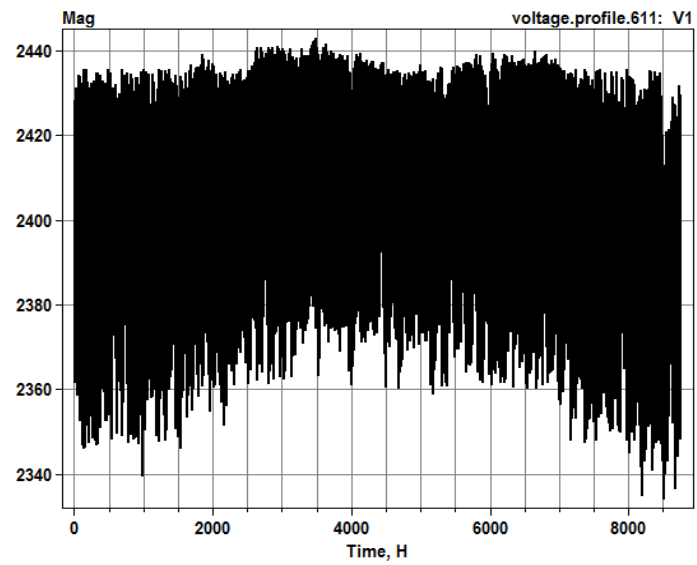

Fig. 9. Voltage profile at node 611 for one year without fault

For this case study, the voltage drop is $2.05 \%$ and the SAIFI is 0.333 . There are less interruptions for this case.

\subsection{Case with fault}

Figure 10 presents the power flow with fault.

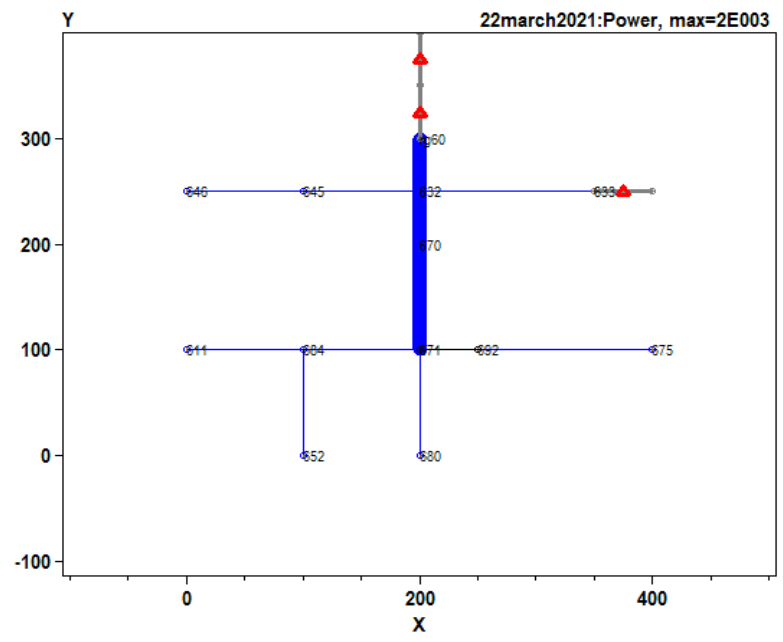

Fig. 10. Power flow with fault

As we observe, the main line is affected by this fault which disturbs the power flow.

Figure 11 shows the voltage profile in the system with fault. We observe that customers can be interrupted if the fault is permanent. There is a vital necessity to integrate Distributed Generation units to limit the effect of this fault along the network. 


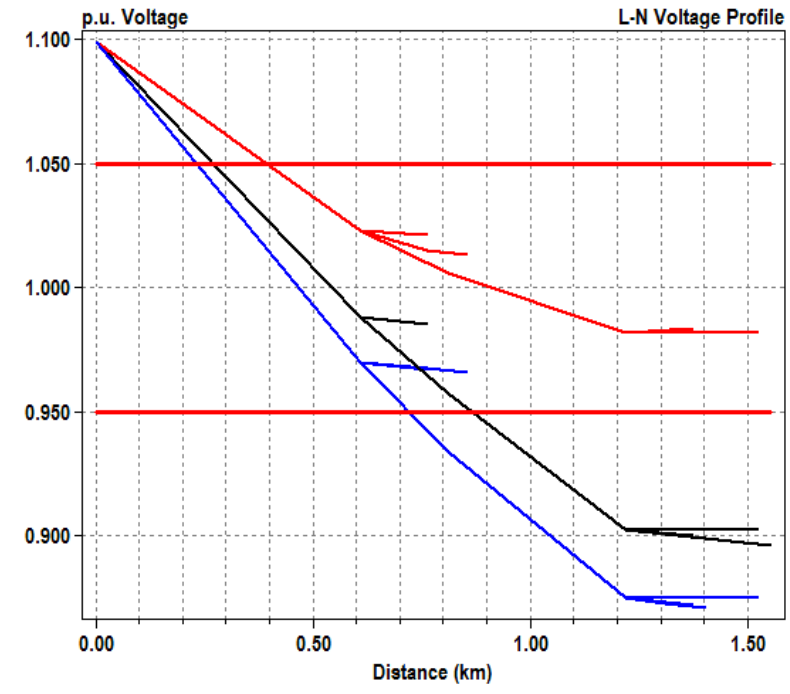

Fig. 11. Voltage profile in the system with fault

Figure 12 presents the voltage profile at node 611 with fault for one year. There is a significant reduction of voltage at loads which are most distanced from substation.

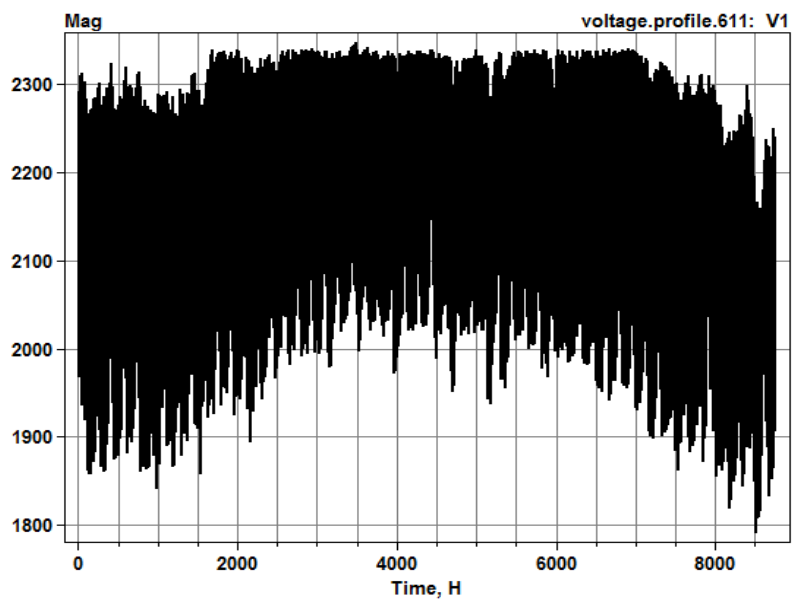

Fig. 12. Voltage profile at node 611 with fault for one year

For this case, the voltage drop is $45 \%$ and the SAIFI is 40. There are many interruptions for this scenario.

\subsection{Case with fault and DGs}

The distributed generation units are located at nodes 632, 670 and 671 with size $150 \mathrm{kVA}, 210 \mathrm{kVA}$ and $250 \mathrm{kVA}$ respectively. Figure 13 presents the power flow with fault and optimum allocation of DGs.

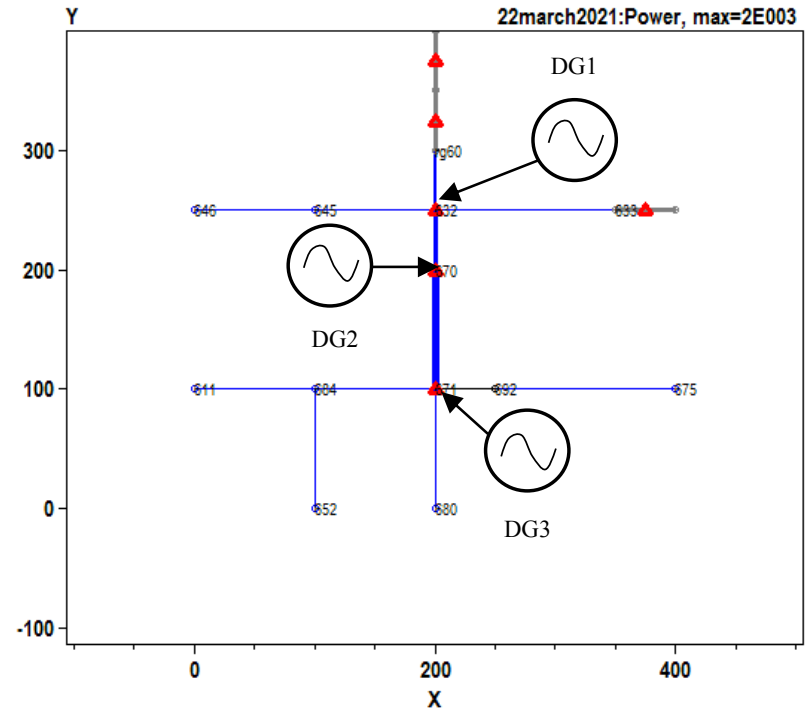

Fig. 13. Power flow with fault and optimum allocation of DGs

Figure 14 presents the voltage profile in the system with fault and DGs. As it is observed, the impact of fault is highly minimized including Distributed Generation units.

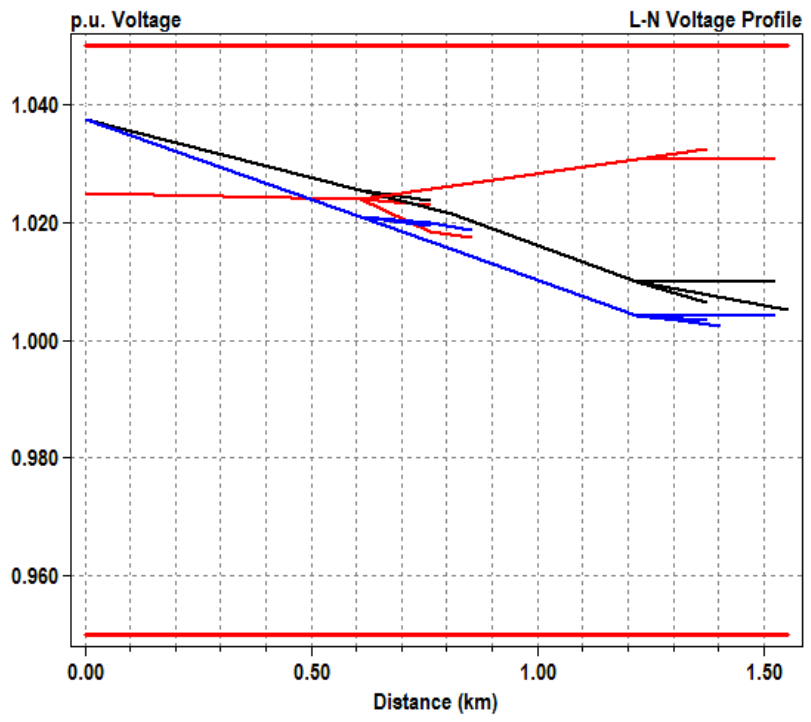

Fig. 14. Voltage profile in the system with fault and DGs

Figure 15 gives the voltage profile at node 611 with fault and DGs for one year. There is an improvement of voltage because DGs give energy to customers and ensure a permanent and continuous supply of electricity. The DGs are both important for customers and environment because they don't pollute the nature. The importance for transition on renewable resources is observed with this major result. 


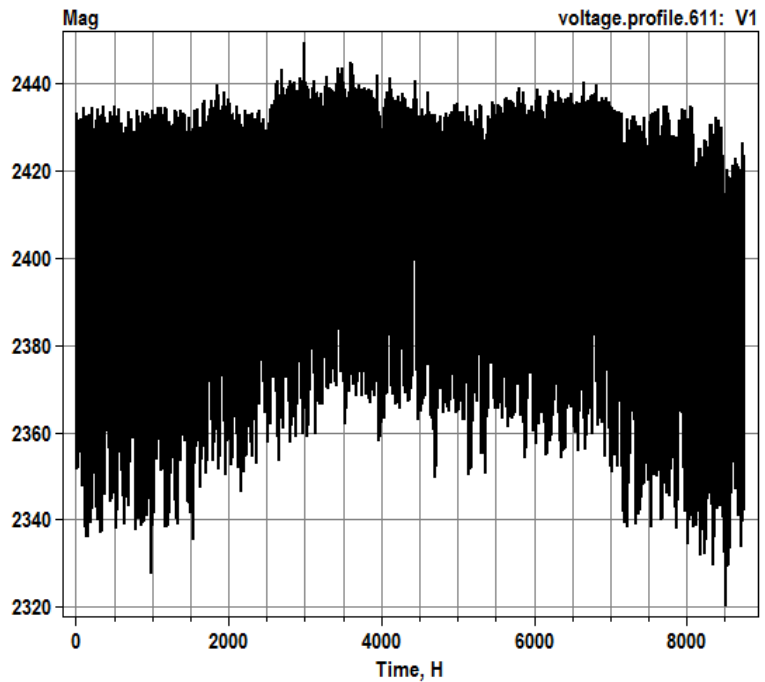

Fig. 15. Voltage profile at node 611 with fault and DGs for one year
For this case, the voltage drop is $2.3 \%$ and the SAIFI is 0.55. The optimum allocation of DGs can reduce the losses and improve the voltage profile with better reliability. This positive effect of distributed generation units justifies the importance for their integration in future electrical networks including new communication technologies.

\subsection{Comparative study}

Figure 16 shows the comparison of system energy consumed for the three test cases for one day. The fault causes an irregular rising of system energy consumed and that can lead to a potential network collapsing. When the DGs are included, the consumption of loads in the system is significantly reduced avoiding the collapse of the system. Figure 17 presents the comparison of energy losses for the three test cases for one day. There is also a major reduction of energy losses with the integration of DGs.

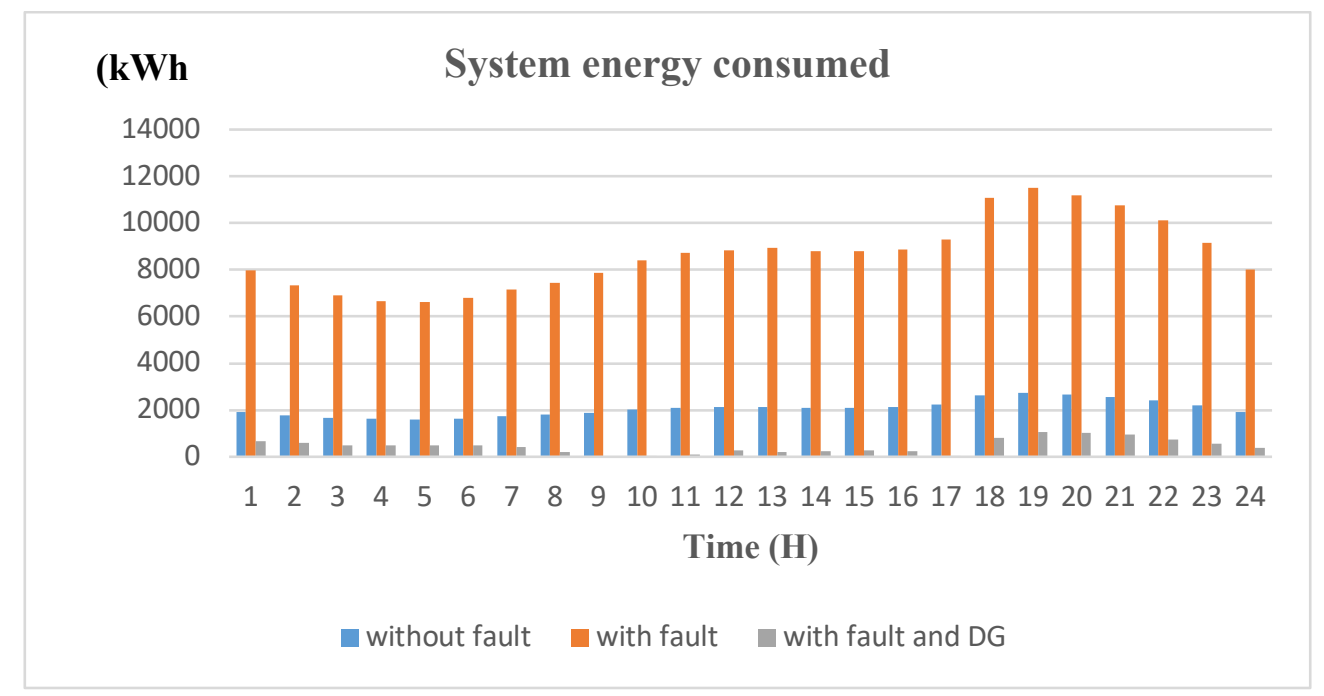

Fig. 16. Comparison of system energy consumed for one day

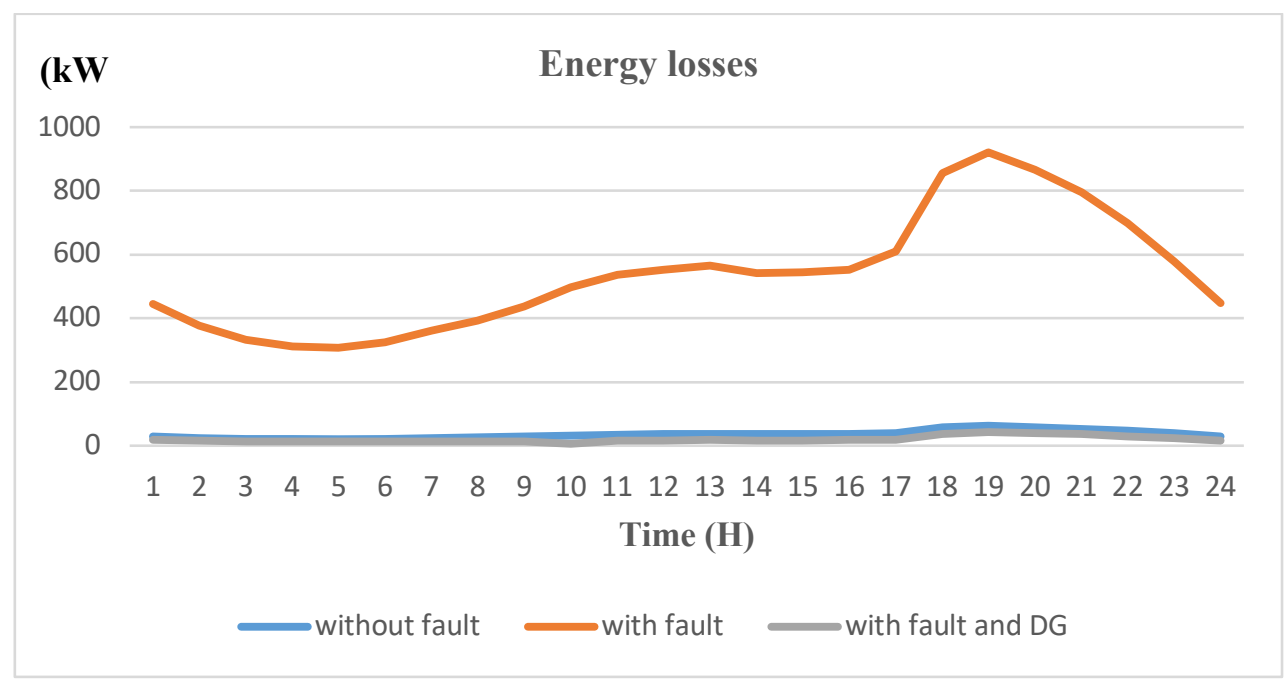

Fig. 17. Comparison of energy losses for one day 
These results show the vital impact of optimum allocation of distributed generations in the power grid.

Table 1 gives a short comparison of our proposed method with the literature.

Table 1. Short comparison with the literature

\begin{tabular}{|c|c|c|}
\hline Method & System & Voltage (pu) \\
\hline Proposed method & $\begin{array}{c}13 \text { node test } \\
\text { feeder }\end{array}$ & 0.99 \\
\hline PSO [13] & $\begin{array}{c}123 \text { node test } \\
\text { feeder }\end{array}$ & 0.97 \\
\hline MLPSO [14] & $\begin{array}{c}33 \text { node test } \\
\text { feeder }\end{array}$ & 0.95 \\
\hline
\end{tabular}

The authors in [11-16] have integrated multiple DGs in conventional network using diverse methods by single simulations. The accuracy of the methods in literature is not significant, it is impossible to control the power flow in the grid, it is also impossible to automate the protective devices in case of fault and to automate the switch with the aim to automatically integrate DGs. The technics in literature don't give a good result in term of loss reduction and reliability optimization because the network is not smart. So, it is important the turn the grid into smart grid to ensure a secure, reliable and smart energy. Our method to co-simulate the smart grid and to include the DGs with smart and optimal allocation give better result comparing with the literature.

\section{Conclusion}

In this work, a novel technic concerning the smart grid co-simulation for reliability issues is proposed. So, the optimum distributed generation allocation and sizing has been applied to enhance the reliability of a smart distribution grid by reducing active losses and improving voltage profile. The proposed OpenDSS-OMNet++ co-simulation is used to achieve the smart grid. The proposed PSO method with OpenDSS and Matlab program is used for the optimization problem in order to make an optimum allocation and sizing of distributed generations in the aim of reducing power losses, improving the voltage profile and enhance reliability. To validate the efficiency of the method, the 13-node IEEE system is simulated for three test cases. The voltage drop with fault is $45 \%$ and the SAIFI is 40 . For the scenario of fault with DG, the voltage drop is $2.3 \%$ and the SAIFI is 0.55 . This satisfactory result shows the efficiency of the proposed co-simulation and PSO algorithm to optimize the reliability of smart grid and the importance to use distributed generation units in the electrical network.

\section{Acknowledgements}

The authors would like to gratefully acknowledge the electrical engineering department of ENSET of Douala for the availability of the laboratory and the necessary devices.

\section{References}

[1] H. Farhangi, "The path of the smart grid", IEEE Power and Energy Mag, pp. 18-28, 2010.

[2] H. Zhang, "Smart Grid Technologies and Implementations", $\mathrm{PhD}$ Thesis, University of London, 2014.

[3] I. Colak, "Introduction to Smart Grid", 3rd International Smart Grid Workshop and Certificate Program (ISGWCP), pp. 21-25, 2016.

[4] A. A. Bani, A. Nasiri, I. Stamenkovic, "Foundational Support Systems of the Smart Grid: State of the Art and Future Trends", International Journal of Smart Grid, Vol. 2, No. 1, pp. 1-12, 2018.

[5] A. Harris, "Smart Grid Thinking”, Engineering \& Technology Magazine, Vol. 4, No. 9, pp. 46-49, 2009.

[6] DRAFT NIST Framework and Roadmap for Smart Grid Interoperability Standards, Release 4.0, www.nist.gov (last accessed 10/03/2021)

[7] K. E. Okedu, W. Z. AL Salmani, "Smart Grid Technologies in Gulf Cooperation Council Countries: Challenges and Opportunities", International Journal of Smart Grid (IJS), Vol. 3, No. 2, pp. 1-11, 2019.

[8] G. Pepermans, J. Driesen, D. Haeseldonckx, R. Belmans, W. Dhaeseleer, "Distributed generation: definition, benefits and issues", Energy policy, Vol. 33, No. 6, pp. 787-798, 2005.

[9] F. Ayadi, I. Colak, I. Garip, H. I. Bulbul, "Impacts of Renewable Energy Resources in Smart Grid", $20208^{\text {th }}$ international conference on Smart Grid (icSmartGrid), pp. 183-188, 2020.

[10] J. P. Lopes, N. Hatziargyriou, J. Mutale, P. Djapic, N. Jenkins, "Integrating distributed generation into electric power systems: A review of drivers, challenges and opportunities", Electric power systems research, Vol. 77, No. 9, pp. 1189-1203, 2007.

[11] J. H. Teng, T. S. Luor, Y. H. Liu, "Strategic Distributed Generator Placements for Service Reliability Improvements", IEEE Power Engineering Society Summer Meeting, Vol. 2, pp. 719-724, 2002.

[12] S. Bhullar, S. Ghosh, "Optimal Integration of Multi Distributed Generation Sources in Radial Distribution Networks Using a Hybrid Algorithm", Energies, pp. 115,2018 .

[13]A. Anwar, H. R. Pota, "Loss Reduction of Power Distribution Network Using Optimum Size and Location of Distributed Generation", Australasian Universities Power Engineering Conference-IEEE, pp. 1-6, 2011. 
[14]E. Karunarathne, J. Pasupuleti, J. Ekanayake, D. Almeida, "Optimal Placement and Sizing of DGs in Distribution Networks Using MLPSO Algorithm", energies, Vol. 13, pp. 1-25, 2020.

[15]P. Parreira, A. Rosentino, D. B. Rodrigues, M. R. Castillo, F. Moura, M. Mendonça, N. Tolentino, R. Rimoldi, W. Baunier, S. Borges, G. B. Lima, "Influence analysis of photovoltaic and energy storage systems in a distribution system in the context of permanent regime Voltage using the OpenDSS", 18th International Conference on Renewable Energies and Power Quality (ICREPQ), pp. 1-6, 2020.

[16] J. C. Mendieta, "Intelligent distribution voltage control with distributed generation", $\mathrm{PhD}$ Thesis, University of Quebec, 2016.
[17] C. Mbey, A. T. Boum, L. Nneme, "Contribution to the Transformation of the Distribution Network of the City of Douala into a Smart Grid using OpenDSS and OMNet++: Reduction of Technical Losses and Improvement of Voltage Profile", International Journal of Engineering Research \& Technology (IJERT), Vol. 9, No. 11, pp. 170-175, 2020.

[18] www. SourceForge.net (last accessed on 10/04/2021)

[19]R. A. Pegado, Y. P. M Rodriguez, "Distribution Network Reconfiguration with the OpenDSS using Improved Binary Particle Swarm Optimization", IEEE Latin America Transactions, Vol. 16, No. 6, pp. $1677-$ 1683, 2018.

[20]L. Chang-Gun, "OMNet++ Tutorial”, School of Computer Science and Engineering, University of Seoul, 2018. 